\title{
Meta-analysis indicating that high ALCAM expression predicts poor prognosis in colorectal cancer
}

\author{
Yeqing Zhang ${ }^{1, *}$, Chunmei Qian ${ }^{2, *}$, Lin Jing ${ }^{3}$, Jianlin Ren ${ }^{3}$ and Yu Guan ${ }^{2}$ \\ ${ }^{1}$ Department of Chinese Internal Medicine, Shanghai Municipal Hospital of Traditional Chinese Medicine, Shanghai University \\ of Traditional Chinese Medicine, Shanghai 200071, China \\ ${ }^{2}$ Department of Central Laboratory Medicine, Shanghai Municipal Hospital of Traditional Chinese Medicine, Shanghai \\ University of Traditional Chinese Medicine, Shanghai 200071, China \\ ${ }^{3}$ Department of Oncology, Shanghai Municipal Hospital of Traditional Chinese Medicine, Shanghai University of Traditional \\ Chinese Medicine, Shanghai 200071, China \\ *These authors contributed equally to this work \\ Correspondence to: Yu Guan, email: newera2017@163.com \\ Jianlin Ren, email: renjanlin666@126.com \\ Keywords: ALCAM, outcome, clinical features, CRC
}

Received: November 30, $2016 \quad$ Accepted: April 24, $2017 \quad$ Published: May 08, 2017

Copyright: Zhang et al. This is an open-access article distributed under the terms of the Creative Commons Attribution License 3.0 (CC BY 3.0), which permits unrestricted use, distribution, and reproduction in any medium, provided the original author and source are credited.

\section{ABSTRACT}

\begin{abstract}
Activated leukocyte cell adhesion molecule (ALCAM) has been linked to the development and progress of colorectal cancer (CRC). In this meta-analysis, we examined whether ALCAM expression is predictive of survival outcomes in CRC patients. We included 7 studies with 2048 patients in our meta-analysis after searching the PubMed, Cochrane Library, EMBASE, OVID and Web of Science databases. High ALCAM expression was associated with poor overall survival among CRC patients $(\mathrm{HR}=1.94,95 \% \mathrm{CI}=1.05-3.58, P=0.03)$. High ALCAM expression was also associated with aggressive clinicopathological features such as tumor stage (T3,T4/ $\mathrm{T1}$, T2; HR $=2.66,95 \% \mathrm{CI}=2.01-3.51, P<0.00001$ ), nodal status (Positive/ Negative, $\mathrm{HR}=2.12,95 \% \mathrm{CI}=1.61-2.82, P<0.00001)$, distant metastasis $(\mathrm{M1} / \mathrm{MO}, \mathrm{HR}=3.30$, $95 \% \mathrm{CI}=2,21-4.91, P<0.00001)$, tumor grade (grade3 $/$ grade1, $2, \mathrm{HR}=1,28,95 \%$ $\mathrm{CI}=1.00-1.62, P=0.05)$, and patient age $(>60 /<60, \mathrm{HR}=1.29,95 \% \mathrm{CI}=1.01-1.66$, $P=0.05)$. These findings indicate that high ALCAM expression is associated with poor prognosis and advanced clinicopathological characteristics in CRC patients.
\end{abstract}

\section{INTRODUCTION}

Colorectal cancer (CRC) is one of most frequently malignant tumors globally, and only $10-20 \%$ of CRC patients are diagnosed early stage [1]. The incidence of $\mathrm{CRC}$ has been increasing rapidly and the treatment is extremely limited for advanced CRC [2]. The conventional prognostic factors for CRC are histological tumor grades and disease stages including cell differentiation, depth of tumor invasion, and lymph node metastasis [3, 4]. In recent studies, cell surface markers were identified as potential therapeutic targets [5-7]. Yet, the prognostic value of these putative molecular biomarkers in CRC was ambiguous. Therefore, there is an urgent need to develop molecular diagnostic markers for CRC that would enable effective and early clinical screening and prevention strategies.
Activated leukocyte cell adhesion molecule (ALCAM or CD166) that belongs to the cell surface immunoglobulin superfamily was first described as a CD6 ligand. The ALCAM gene is mapped to human chromosome 3q13 [8,9]. CD166 is usually expressed in proliferative or trafficking cells like activated leukocytes, embryonic hematopoietic and endothelial cells, lung endothelial cells, endometrial cells, and blastocysts [10]. Modulation of CD166 function inhibits matrix metalloproteinase-2 activation [11], which decreases tumor angiogenesis and the expression of extracellular matrix proteins, thereby altering tumor progression [12]. Also, ALCAM expression has been reported in prostate [13], breast [14], ovarian [15], pancreatic [16], and colorectal [17] cancers. Although many studies have reported the prognostic value of ALCAM expression in CRC $[18,19]$, 
the results have been contradictory. This may have been caused by limited study samples in the studies in addition to other factors. Therefore, we conducted a pooled analysis to investigate the clinical significance of ALCAM in CRC and its potential value as a biomarker.

\section{RESULTS}

\section{Study selection}

We retrieved 107 studies after comprehensively searching PubMed, Cochrane Library, EMBASE, OVID, Web of Science databases and other sources. Two duplicate reports were excluded. After detailed screening by title and abstracts, 86 more records were excluded. A full-text assessment of the remaining 21 articles resulted in exclusion of 14 more studies and finally 7 studies that fulfilled the inclusion criteria were enrolled in our metaanalysis [19-25]. A flow chart of the selection process is shown in Figure 1.

\section{Study characteristics}

Table 1 shows details of the 7 studies included in the meta-analysis. A total of 2048 CRC patients were included in this meta-analysis. Among the 7 studies, 3 were from Germany and 2 each from Korea, Australia and Switzerland. The ALCAM expression was measured by tissue microassay (TMA) or immunohihstochemistry (IHC) in the 7 studies. The studies also included overall survival (OS) and disease-free survival (DFS) statistics.

\section{Quality assessment of studies}

Table 2 shows the quality assessment of the 7 included studies by the Newcastle-Ottawa Scale (NOS) grading system taking into account selection, comparability and outcome of each study. The NOS scores for the 7 studies ranged from 6 to 9 and therefore regarded as high quality.

\section{Association between ALCAM expression and OS}

The random-effects model was used to analyze the prognostic value of ALCAM expression in CRC based on heterogeneity test results $\left(\mathrm{I}^{2}=85 \%, P<0.00001\right)$. Higher ALCAM expression was associated with poor survival in CRC patients $(\mathrm{HR}=1.94,95 \% \mathrm{CI}=1.05-3.58$, $P<0.00001$; Figure 2). Figure 3 shows results of subgroup analysis based on five stratifications, namely, survival parameters, ethnicity, testing methods, staining pattern and follow-up time. Our analysis showed that high ALCAM expression was associated with poor overall survival of CRC patients $(\mathrm{HR}=1.47,95 \% \mathrm{CI}=1.12-1.92$, $P<0.00001)$. However, no significant association was observed in disease-free survival (DFS) group. Further, our results showed that studies using IHC to detect ALCAM expression predicted poorer outcomes of CRC patients $(\mathrm{HR}=3.07,95 \% \mathrm{CI}=1.97-4.76, P=0.0002)$ compared to studies using tissue microarray (TMA) to estimate ALCAM levels ( $\mathrm{HR}=1.01,95 \% \mathrm{CI}=0.78-1.30, P=0.45)$. Regarding ethnicity, high ALCAM expression predicted poor prognosis of Asian CRC patients (HR $=3.52$, $95 \% \mathrm{CI}=2.05-6.06, P<0.00001)$. Moreover, high ALCAM expression was associated with membrane and cytoplasmic staining $(\mathrm{HR}=3.27,95 \% \mathrm{CI}=2.16-4.94$, $P=0.002)$. No significant association was observed between ALCAM expression and CRC patients based on follow-up time. The results of overall and subgroup analysis are summarized in Supplementary Table 1.

Among the 7 included studies, 5 focused on overall survival (OS) of patients and 2 on disease-free survival. Subgroup analysis on the 5 studies that focused on overall survival based on testing methods, ethnicity, staining pattern and follow-up time revealed that high expression of ALCAM predicted poor prognosis of CRC patients in membrane and cytoplasmic staining $(\mathrm{HR}=3.0,95 \% \mathrm{CI}=$ $1.92-4.69, P<0.00001)$, IHC method (HR $=2.75,95 \% \mathrm{CI}$ $=1.70-4.44, P<0.00001)$, Asian ethnicity $(\mathrm{HR}=3.06$, $95 \% \mathrm{CI}=1.65-5.69, P<0.00001)$ and $\geq 50(\mathrm{HR}=2.06$, $95 \% \mathrm{CI}=1.22-3.48, P<0.00001$ ) groups (Supplementary Figure 7; Supplementary Table 1). However, these results were limited by smaller sample sizes when the subjects were divided into subgroups. However, there was no heterogeneity among the 5 studies and therefore we predict that our results were accurate.

\section{Correlation of ALCAM to clinicopathological features}

Next, we analyzed the relationship between ALCAM expression and clinical features of CRC like tumor stage, nodal status, distant metastasis, tumor grade, age and gender. Six studies reported ALCAM expression in CRC patients with clinical tumor stages and our analysis showed that ALCAM overexpression was associated with advanced tumor stage [pooled OR $(\mathrm{T} 3, \mathrm{~T} 4$ vs. T1,T2) $=2.66$, $95 \% \mathrm{CI}=2.01-3.51, P<0.0001$; Figure 4). Moreover, higher ALCAM expression predicted positive nodal status in CRC patients $(\mathrm{HR}=2.12,95 \% \mathrm{CI}=1.61-2.82$, $P<0.0001$; Supplementary Figure 1). High expression of ALCAM was also significantly associated with distant metastasis $(\mathrm{HR}=3.30,95 \% \mathrm{CI}=2.21-4.91, P<0.0001$; Supplementary Figure 2). Analysis of 5 studies with 1826 cases that reported significance of ALCAM expression to tumor grade revealed that ALCAM overexpression was associated with the higher tumor grade $(\mathrm{HR}=1.28$, $95 \% \mathrm{CI}=1.00-1.62, P=0.05$; Supplementary Figure 3). Stratification analysis also showed association of high ALCAM expression with higher patient age and the poor risk of differentiation $(\mathrm{HR}=1.29,95 \% \mathrm{CI}=1.01-1.29$, $P=0.05$; Supplementary Figure 4). Although ALCAM 
Table 1: Baseline characteristics of the studies included

\begin{tabular}{|c|c|c|c|c|c|c|c|c|c|c|}
\hline No. & First Author & Year & Country & Sample size & $\begin{array}{l}\text { Mean Age } \\
\text { (Year) }\end{array}$ & $\begin{array}{c}\text { Duration of } \\
\text { Follow- } \\
\text { up(month) }\end{array}$ & $\begin{array}{c}\text { Survival } \\
\text { Conditions }\end{array}$ & $\begin{array}{l}\text { Testing } \\
\text { Methods }\end{array}$ & $\begin{array}{l}\text { Staining } \\
\text { Pattern }\end{array}$ & $\operatorname{RR}(95 \% \mathrm{CI})$ \\
\hline 1 & Jerry Zhou & 2015 & Australia & 45 & 64 & NM & OS & TMA & $\begin{array}{c}\text { Membrane } \\
\text { and cytoplasm } \\
\text { staining }\end{array}$ & $5.26(1.57-17.62)$ \\
\hline 2 & Michael Tachezy & 2012 & Germany & 300 & $54(15-91)$ & $39(1-180)$ & OS & TMA & $\begin{array}{l}\text { Membrane } \\
\text { staining }\end{array}$ & $0.61(0.40-0.93)$ \\
\hline 3 & Sung Hoon Sim & 2014 & Korea & 112 & $62(33-82)$ & 48.1 & DFS & $\mathrm{IHC}$ & $\begin{array}{c}\text { Membrane } \\
\text { and cytoplasm } \\
\text { staining }\end{array}$ & $5.61(1.82-17.36)$ \\
\hline 4 & A Lugli & 2010 & Switzerland & 1274 & $69.9(30-96)$ & 56.4 & DFS & TMA & $\begin{array}{l}\text { Membrane } \\
\text { staining }\end{array}$ & $0.85(0.56-1.29)$ \\
\hline 5 & Hee Jin Lee & 2013 & Korea & 96 & 67 & NM & OS & $\mathrm{IHC}$ & $\begin{array}{c}\text { Membrane } \\
\text { and cytoplasm } \\
\text { staining }\end{array}$ & $3.06(1.65-5.69)$ \\
\hline 6 & W Weichert & 2004 & Germany & 111 & $65(41-87)$ & 47 & OS & $\mathrm{IHC}$ & $\begin{array}{c}\text { Membrane } \\
\text { and cytoplasm } \\
\text { staining }\end{array}$ & $2.34(1.10-4.98)$ \\
\hline 7 & David Horst & 2009 & Germany & 110 & $66.5(41-92)$ & $94.8(4.8-162)$ & OS & TMA & $\begin{array}{l}\text { Membrane } \\
\text { staining }\end{array}$ & $2.06(1.22-3.48)$ \\
\hline
\end{tabular}

Abbreviations: NM: not mentioned; TMA: tissue microassay; IHC: immunohistochemistry; OS: overall survival; DFS: disease-free survival.

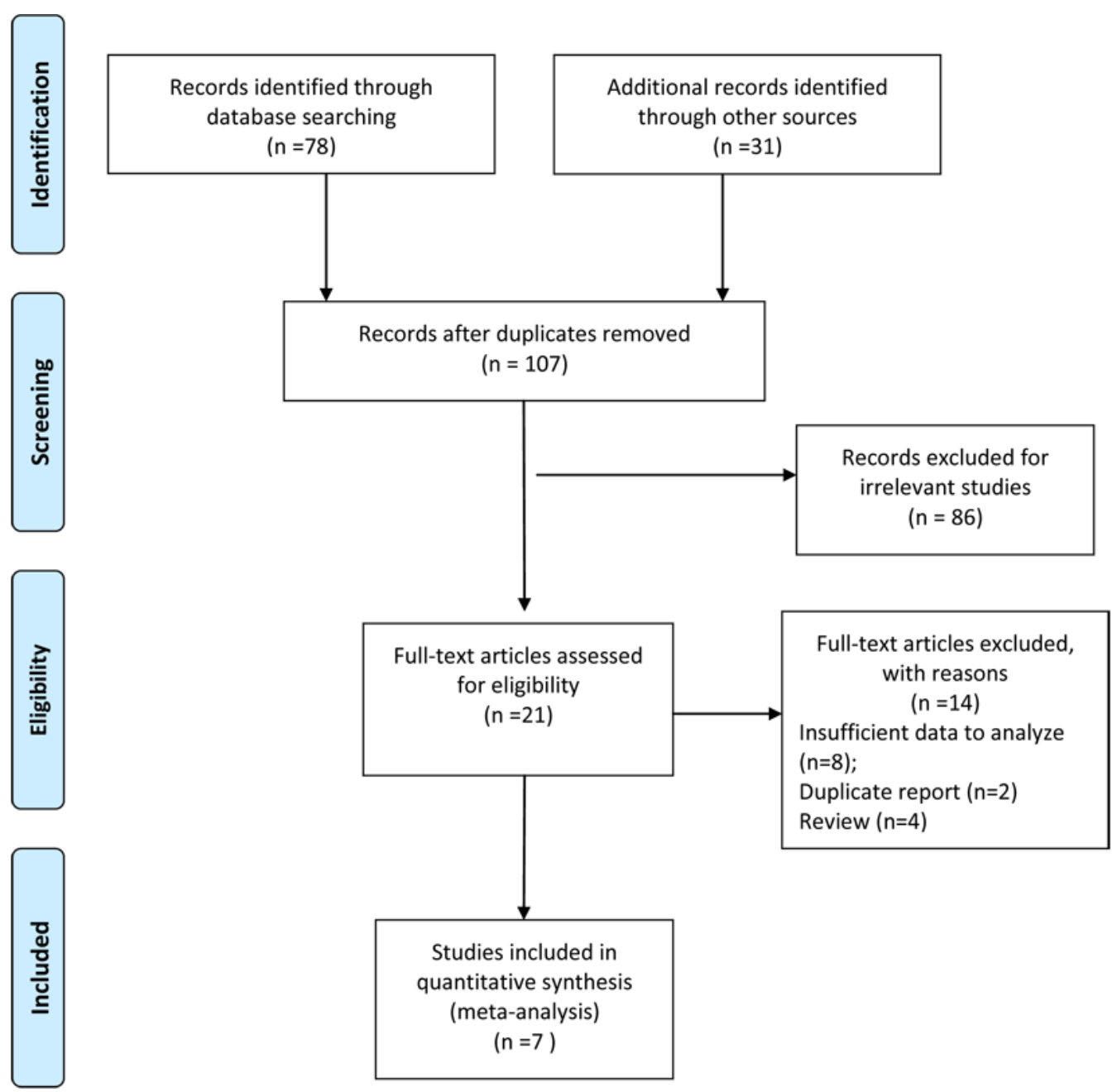

Figure 1: Flow diagram of the study selection process. 
Table 2: Quality assessment using the newcastle-ottawa quality assessment scale in the studies

\begin{tabular}{|c|c|c|c|c|c|c|c|c|c|}
\hline \multirow{2}{*}{ Study (Author, years) } & \multicolumn{3}{|c|}{ Selection } & \multirow[b]{2}{*}{4} & \multirow{2}{*}{ Comparability } & \multicolumn{3}{|c|}{ Outcome } & \multirow{2}{*}{ Score } \\
\hline & 1 & 2 & 3 & & & 1 & 2 & 3 & \\
\hline Jerry Zhou (2015) & $\star$ & $\star$ & $\star$ & - & $\star \star$ & $\star$ & $\star$ & - & 7 \\
\hline Michael Tachezy (2012) & $\star$ & $\star$ & - & $\star$ & $\star \star$ & $\star$ & $\star$ & $\star$ & 8 \\
\hline Sung Hoon Sim 2014) & $\star$ & $\star$ & - & $\star$ & $\star$ & $\star$ & - & $\star$ & 7 \\
\hline A Lugli (2010) & $\star$ & $\star$ & $\star$ & $\star$ & $\star \star$ & $\star$ & - & - & 7 \\
\hline Hee Jin Lee (2013) & - & $\star$ & $\star$ & - & $\star \star$ & - & $\star$ & $\star$ & 6 \\
\hline W Weichert (2004) & $\star$ & $\star$ & $\star$ & $\star$ & $\star \star$ & $\star$ & $\star$ & $\star$ & 9 \\
\hline David Horst (2009) & $\star$ & - & $\star$ & $\star$ & $\star \star$ & $\star$ & $\star$ & $\star$ & 8 \\
\hline
\end{tabular}

expression was not associated with gender of patients, male CRC patients had lower ALCAM expression ( $\mathrm{HR}=0.94$, $95 \% \mathrm{CI}=0.69-1.29, P=0.72$; Supplementary Figure 5). These results are summarized in Supplementary Table 2.

\section{Sensitivity analysis}

We conducted sensitivity analysis to assess the potential heterogeneity between studies by removing one study each time. As shown in Figure 5, no changes were observed in regard to the original meta-analysis suggesting that it was robust and consistent.

\section{Publication bias with trim and filling method}

We evaluated publication bias of the 7 studies using funnel plots (Figure 6), based on Begg's and Egger's test (Figure 7) and observed no obvious asymmetry $(P=0.230)$. Further, we conducted Trim and Filling analysis to robustly analyze publication bias and found that two more studies were necessary to eliminate publication bias thoroughly (variance $=0.578, P=0.194$, Supplementary Figure 6).

\section{Re-sampling statistics}

Next, we used the bootstrap re-sampling procedure to generate 1000 re-sampling groups using overall ORs to estimate the accuracy and robustness of this meta-analysis. Our results demonstrated that the meta-analysis results were robust (pooled $\mathrm{OR}=1.88,95 \% \mathrm{CI}=1.87-1.88$, $P<0.0001$; Supplementary File 1).

\section{DISCUSSION}

Numerous studies have investigated the association between ALCAM expression and the survival outcomes of CRC patients, but, the results have been contradictory. Tachezy et al. demonstrated that the high ALCAM expression was associated with the prolonged survival of CRC patients [21]. However, Weichert et al. reported that high ALCAM expression indicated poor overall survival in univariate analysis, contrary to previous findings [24]. Since these studies indicated the potential prognostic value of ALCAM in CRC despite the contradictory results, we conducted this meta-analysis to clarify the prognostic importance in CRC.

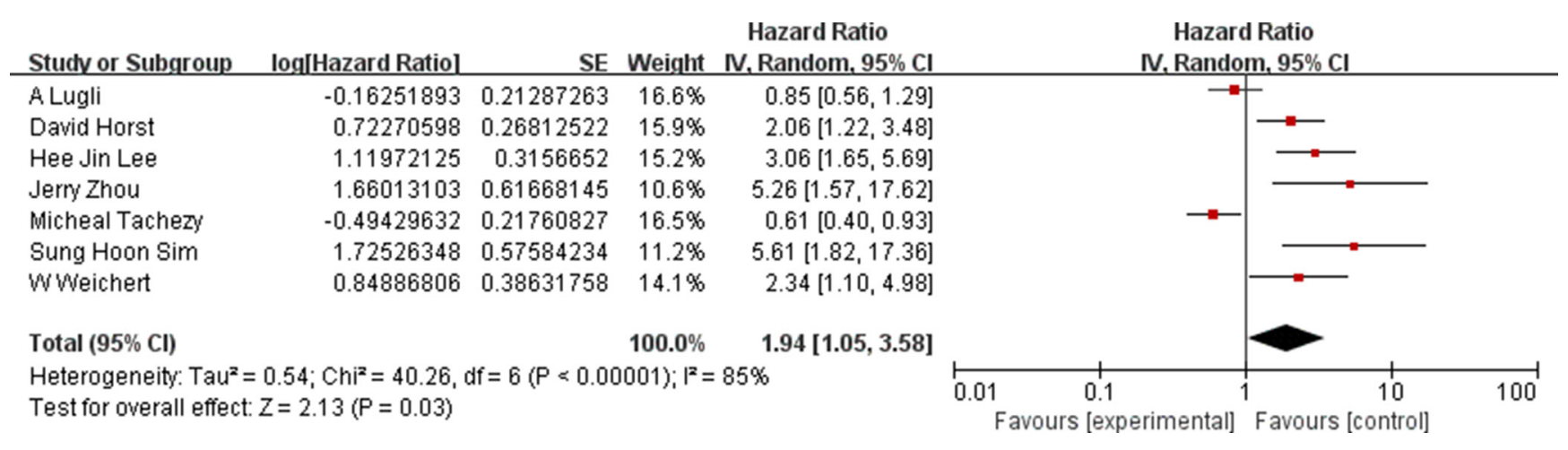

Figure 2: Association between high ALCAM expression and overall survival of CRC patients. 
A

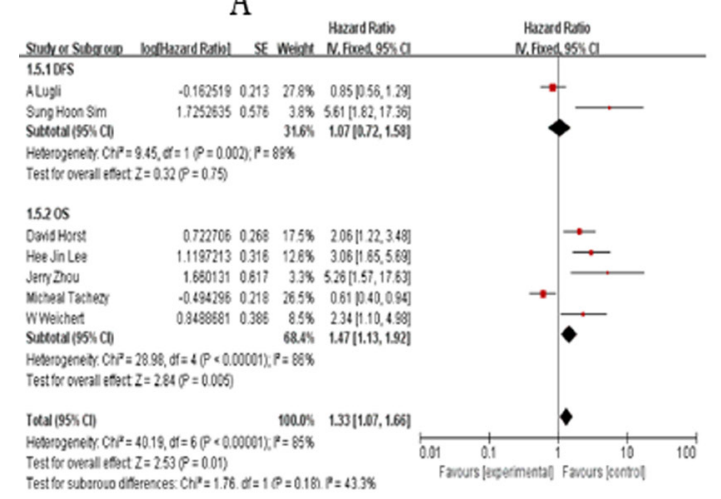

$\mathrm{C}$

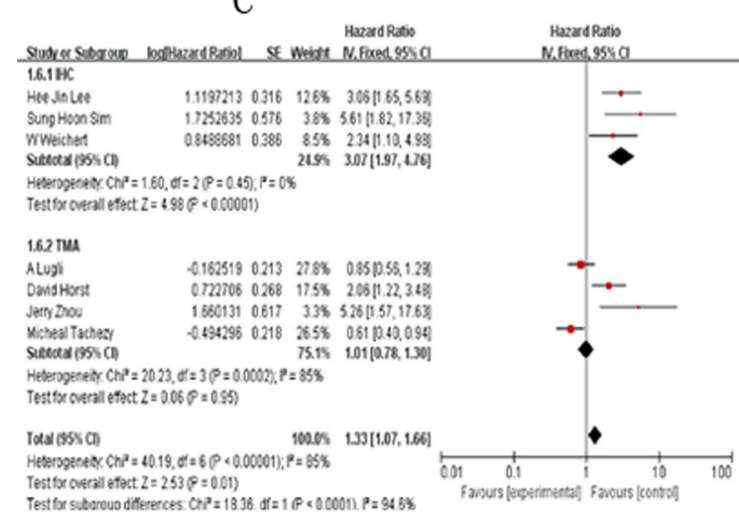

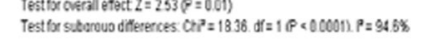

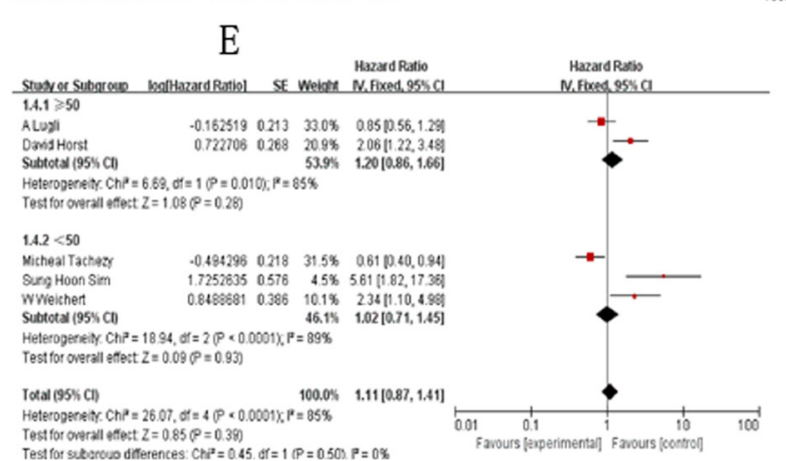

B

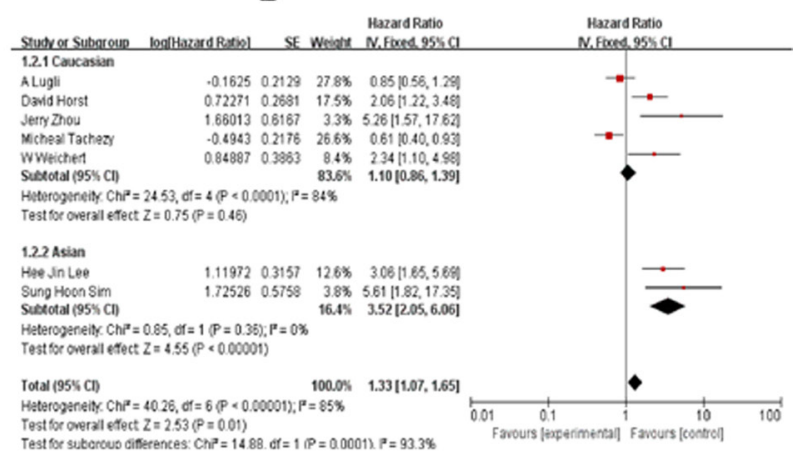

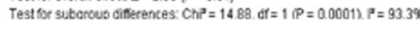

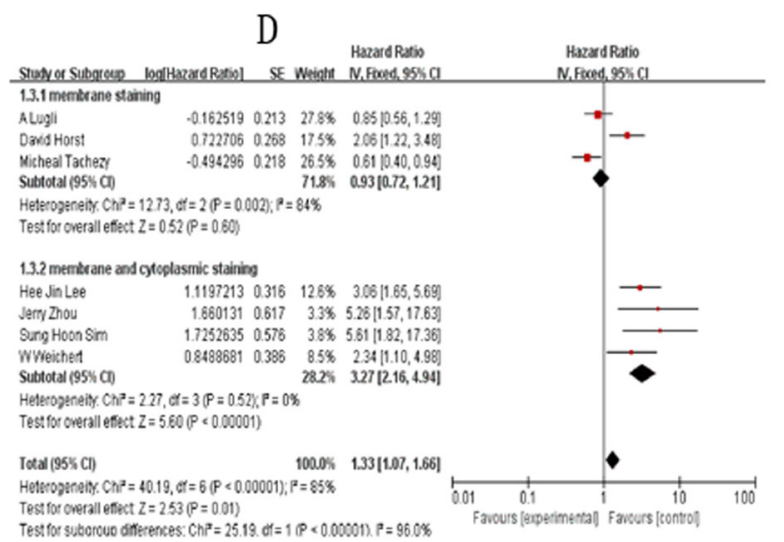

Figure 3: Subgroup analysis results showing association of ALCAM overexpression and overall survival of CRC patients. (A) Results of subgroup analysis based on survival; (B) Results of subgroup analysis based on ethnicity; (C) Results of subgroup analysis based on testing methods; (D) Results of subgroup analysis based on staining pattern; (E) Results of subgroup analysis based on follow-up time.

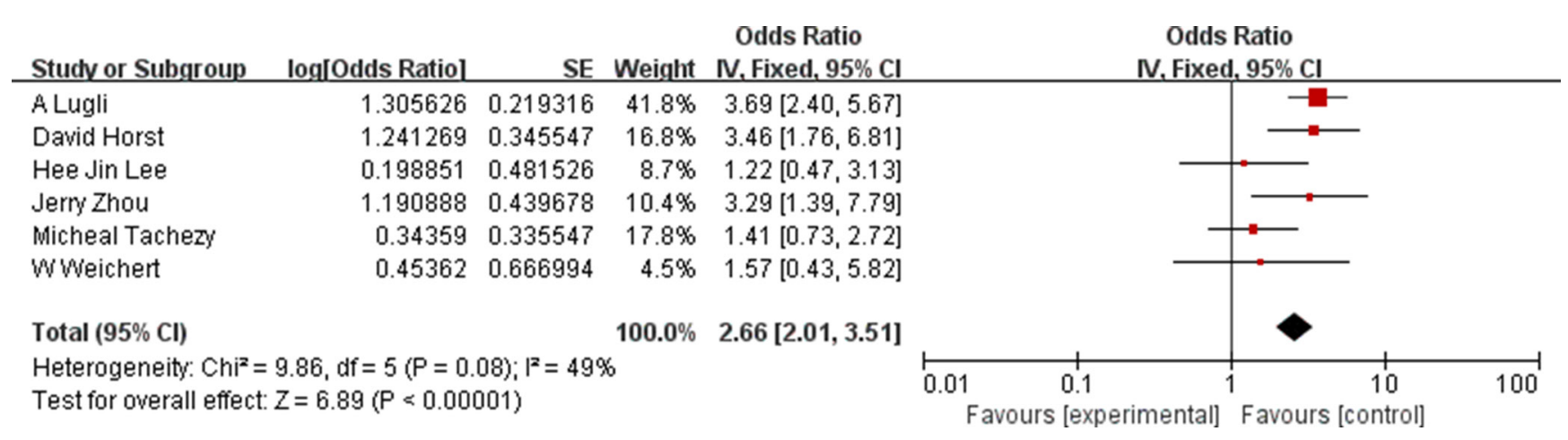

Figure 4: Association between ALCAM overexpression and tumor stage. 
Our data indicated that overexpression of ALCAM predicted poor outcomes for clinical features like nodal status (positive vs. negative), tumor stage (T3, T4 vs. T1, T2), distant metastasis (M1 vs. M0), grade (grade3 vs. grade1,2) and age $(>60$ vs. $<60)$. No correlation was found between ALCAM expression and gender in CRC patients. Also, high ALCAM expression was associated with poor survival outcomes of CRC patients using the random-effects model. Previously, Ni et al. demonstrated the prognostic value of CD166 (ALCAM) expression in digestive cancers [26]. However, only four studies on colorectal cancer (CRC) were enrolled to evaluate the association between ALCAM overexpression and clinical features. Further, the prognostic value of high ALCAM expression in CRC was not investigated [26]. Also, the clinicopathological characteristics and tumor-driving

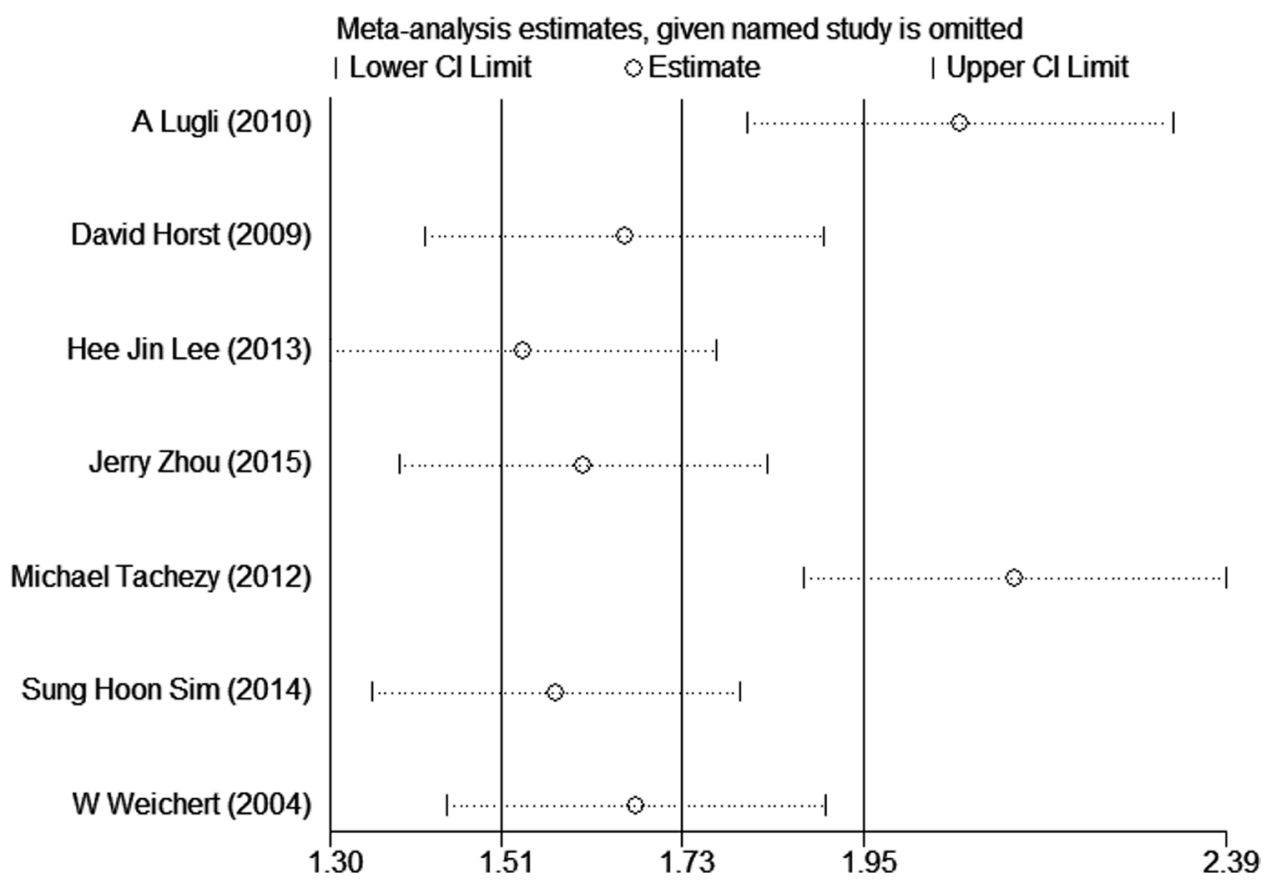

Figure 5: Sensitivity analyses among included studies.

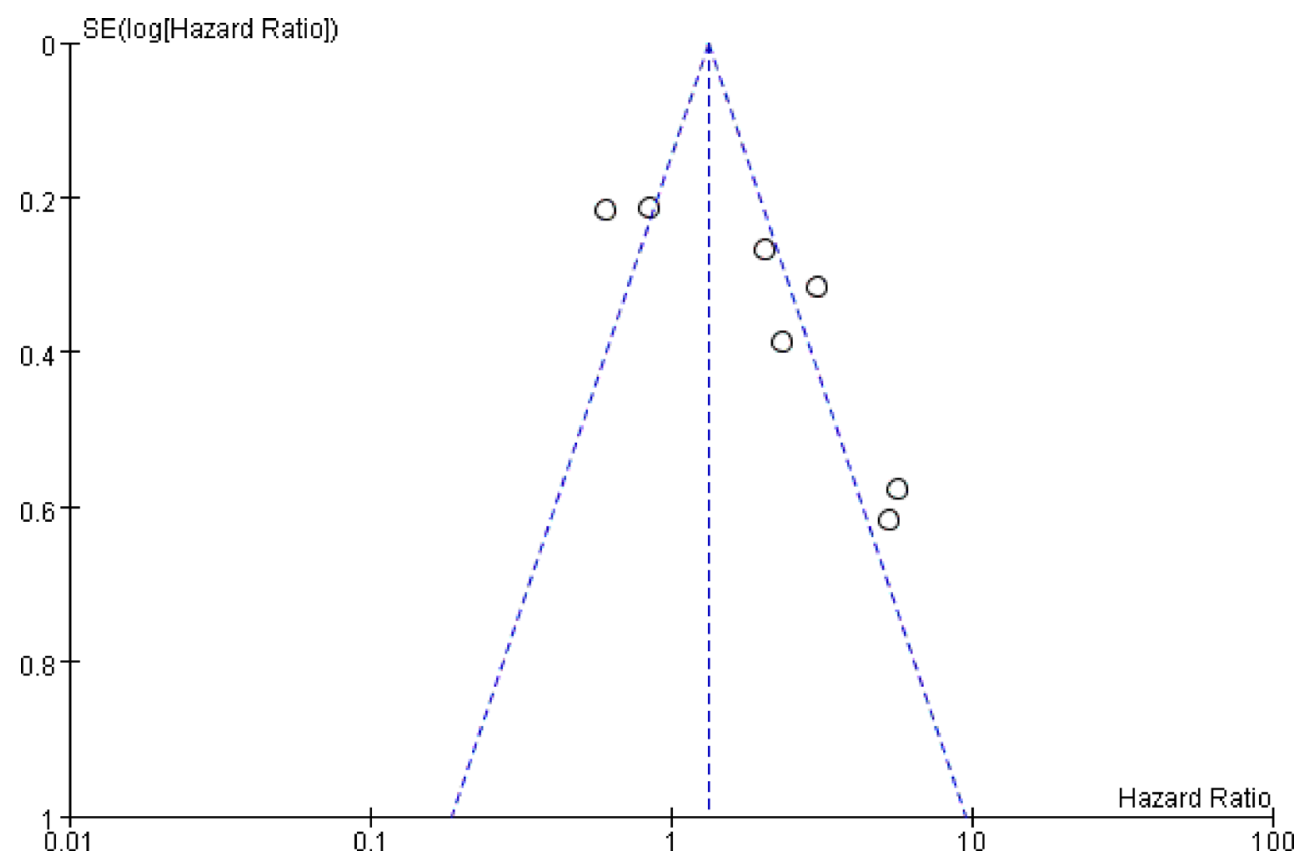

Figure 6: Funnel plot analysis of publication bias between high ALCAM expression and overall survival of CRC patients. 
factors can vary for different types of cancers. Hence, we conducted this meta-analysis to further evaluate the prognostic value of ALCAM high expression in CRC patients. Compared to Ni's study, we systematically retrieved the studies from multiple databases with November 11, 2016 being the last search time. We also comprehensively analyzed the predictive value of ALCAM in evaluating the outcomes of CRC patients in subtypes.

Among the included studies, the staining patterns of ALCAM were highly heterogeneous. Three studies focused on membranous staining [21, 22, 25], whereas the other four studies considered both membrane and cytoplasmic staining. The four studies that analyzed both membrane and cytoplasmic staining indicated that high ALCAM expression reflected poor outcomes of CRC patients. In contrast, no significant association was found between ALCAM expression and clinical outcome by studies analyzing membrane staining only. Interestingly, in breast [27], ovarian [15] and oral [28] cancers, high membrane and cytoplasmic ALCAM expression is associated with poor outcomes.

Another source of heterogeneity in our analysis was the detecting methods of ALCAM expression. Three studies used immunohistochemistry and showed that high ALCAM expression indicated poor prognosis. These results were consistent with our findings. However, the 4 studies that used tissue microarray to analyze ALCAM expression showed no association. This could be because the antibody concentrations used for TMA ranged from $1: 100$ to $1: 500$ making it difficult to determine standard cut-off scores for the positive staining.

Although several molecular tumor markers were reported previously, none were amenable in clinical trials [29]. KRAS mutational analysis remains the only clinical biomarker for CRC [30]. Therefore, new biomarkers such as ALCAM need to be developed for clinical use. However, the mechanistic link between ALCAM and CRC is unknown. ALCAM may influence homotypic and heterotypic interactions between cells [31-33]. However, more mechanistic studies are needed. Besides, ALCAM can be activated by the KRAS mutations, thereby contributing to $\mathrm{CRC}$ tumorigenesis and metastasis [34]. Furthermore, activation of $\beta$-catenin by loss of adenomatous poluposis coil (APC) induces ALCAM expression .

There are some limitations in our meta-analysis. First, inconsistent cut-off scores among studies because of different detecting methods may contribute to heterogeneity. Second, the number of studies enrolled in our analysis is relatively small. Hence, our findings need to be confirmed by larger studies in future. Third, calculation errors could not be avoided when estimating data from Kaplan-Meier curves using Engage Digitizer 4.1. Finally, heterogeneity existed in some pooled outcomes and could not be resolved by subgroup analysis.

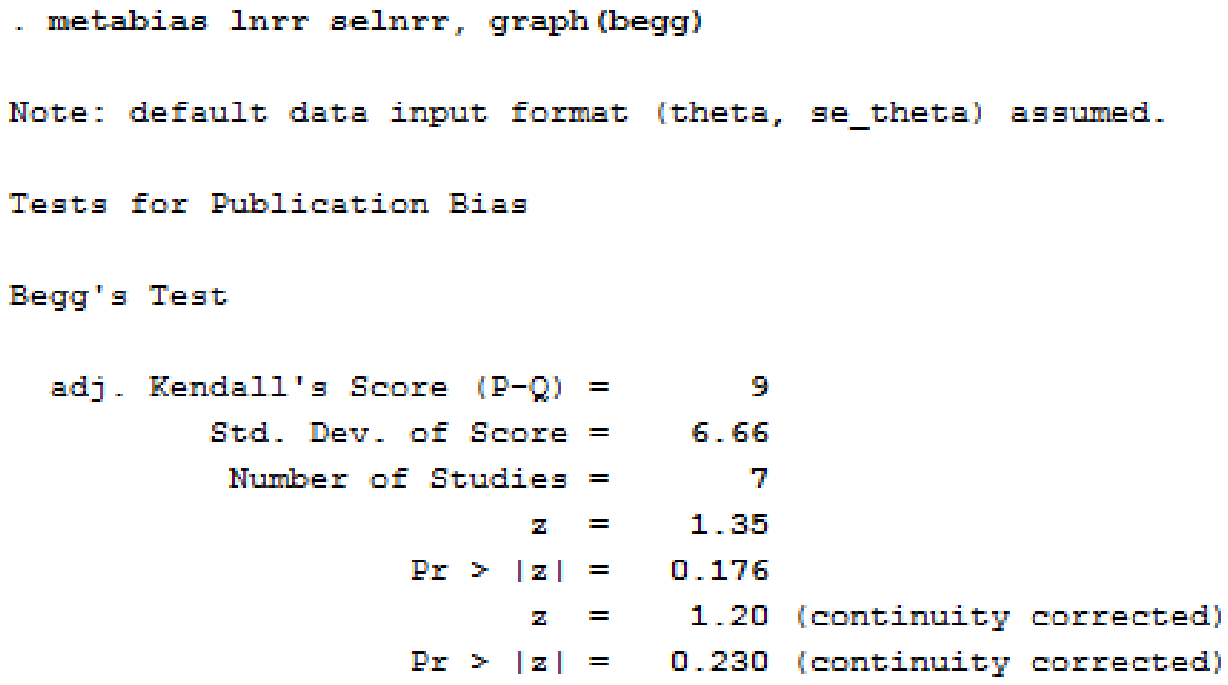

\begin{tabular}{r|rrrrrr}
\hline Std_Eff & Coef. & Std. Brr. & $t$ & P > t l & [958 Conf. Interval] \\
\hline slope & -1.326516 & .5118024 & -2.59 & 0.049 & -2.642146 & -.0108862 \\
bias & 5.779792 & 1.724544 & 3.35 & 0.020 & 1.346709 & 10.21287 \\
\hline
\end{tabular}

Figure 7: Begg's and Egger's tests of publication bias. 
In conclusion, our meta-analysis shows that high expression of ALCAM is associated with poor outcome of $\mathrm{CRC}$ and predicts aggressive clinicopathological features. Therefore, ALCAM is a potential biomarker of clinical relevance for CRC patients.

\section{MATERIALS AND METHODS}

\section{Literature research}

We comprehensively searched the electronic databases PubMed, Cochrane Library, EMBASE, OVID and Web of Science using the following search keywords: ALCAM or CD166 and colorectal cancer or colorectal carcinoma or colon cancer or rectal cancer or CRC. Other bioinformatic sources such as Oncomine and TCGA (analyzed by cBioPortal) were also searched. The searches were conducted until November 11, 2016. The reference lists of all eligible studies were evaluated to avoid missing any potential data. Authors of articles were contacted if specific information was required.

\section{Selection criteria}

The inclusion criteria were: 1) case-control and cohort studies that focused on the association between ACLAM expression and its prognostic significance in colorectal cancer; 2) studies with sufficient data to calculate the odds ratio $(\mathrm{OR})$ or hazard ratio $(\mathrm{HR})$ and the corresponding 95\% CI; 3) the expression level of ALCAM was definitively tested by standard molecular assays; 4) adequate patients and control subjects were enrolled in studies (more than 40).

The exclusion criteria were: 1) letters, duplicate studies, reviews and case reports, 2) studies without sufficient data, 3) animal experiment studies or in vitro cell culture models. When multiple studies focused on the same population, the most recent one was used.

\section{Data extraction}

Two authors (Y. Zhang and C. Qian) extracted the following data independently from the included studies: author names, publication years, ethnicity, country, sample size, mean age, distribution of age and gender, median follow-up time, survival condition, staining patterns, HR estimation and corresponding 95\% CI, tumor node metastases, histological stage, differentiation degree and nodal status. We preferred multivariate analysis if both univariate and multivariate analysis were reported. If the studies provided only the Kaplan-Meier curves, data was extracted with the Engauge Digitizer 4.1 software. The data was thoroughly examined and any discrepancies were resolved by all authors.

\section{Quality assessment}

The Newcastle-Ottawa Scale (NOS) was used to evaluate the quality of studies enrolled in our meta- analysis. The NOS system uses a star system based on three parts: selection (4 stars), comparability (2 stars) and outcome (3 stars). In this meta-analysis, studies that received 6 or more stars out of a maximum of 9 were regarded as high-quality. Any disagreements were resolved by all authors by discussion.

\section{Statistical analysis}

The hazard ratios (HRs) and the corresponding 95\% confidence intervals (CIs) were used to evaluate the prognostic value of ALCAM in colorectal cancer. Odds ratios (ORs) were used to assess the correlation between ALCAM expression and clinicopathological parameters (tumor node metastases, histological stage, differentiation degree and nodal status). Heterogeneity test was conducted to assess heterogeneity between studies using $Q$-test and $\mathrm{I}^{2}$ index. Random-effects or fixed-effects models were used based on the results of heterogeneity test. The statistical analysis was conducted by Review Manager Version 5.3 software (http://ims.cochrane.org/revman). Sensitivity analysis was performed by removing a study each time to assess the stability of our results. Begg's and Egger's asymmetry tests were performed by STATA software version 12.0 to identify potential publication biases in outcomes. Finally, the accuracy of the entities that were calculated in this meta-analysis was estimated by applying the bootstrap re-sampling procedure, which generated 1000 re-sampling groups to get robust and replicable results. Then overall ORs were analyzed for re-sampling statistics.

\section{ACKNOWLEDGMENTS AND FUNDING}

This work was supported by grants from National Natural Sciences Foundation of China. (Grant No. 81500676 to $\mathrm{Yu}$ Guan and 81473628 to Jianlin Ren); Science \& Technology Commission of Shanghai Municipality grants (grant No. 14ZR1438900 to Jianlin Ren) and Outstanding Youth Foundation of Shanghai Municipal Hospital of Traditional Chinese Medicine (Grant No. 20140009 to Yu Guan).

\section{CONFLICTS OF INTEREST}

The authors declare no conflicts of interest.

\section{REFERENCES}

1. El Khoury F, Corcos L, Durand S, Simon B, Le Jossic-Corcos C. Acquisition of anticancer drug resistance is partially associated with cancer stemness in human colon cancer cells. Int J Oncol. 2016; 49:2558-2568. doi: 10.3892/ijo.2016.3725.

2. Sameer AS, Nissar S. Epigenetics in diagnosis of colorectal cancer. Mol Biol Res Commun. 2016; 5:49-57.

3. Boman BM, Huang E. Human colon cancer stem cells: a new paradigm in gastrointestinal oncology. J Clin Oncol. 2008; 26:2828-38. doi: 10.1200/JCO.2008.17.6941. 
4. Dalerba P, Dylla SJ, Park IK, Liu R, Wang X, Cho RW, Hoey T, Gurney A, Huang EH, Simeone DM, Shelton AA, Parmiani G, Castelli C, et al. Phenotypic characterization of human colorectal cancer stem cells. Proc Natl Acad Sci USA. 2007; 104:10158-63. doi: 10.1073/pnas.0703478104.

5. Ricci-Vitiani L, Lombardi DG, Pilozzi E, Biffoni M, Todaro M, Peschle C, De Maria R. Identification and expansion of human colon-cancer-initiating cells. Nature. 2007; 445:111-5. doi: 10.1038/nature05384.

6. Gao L, Bai L, Nan Q. Activation of Rho GTPase Cdc42 promotes adhesion and invasion in colorectal cancer cells. Med Sci Monit Basic Res. 2013; 19:201-7. doi: 10.12659/ MSMBR.883983.

7. Jordan CT, Guzman ML, Noble M. Cancer stem cells. $\mathrm{N}$ Engl J Med. 2006; 355:1253-61. doi: 10.1056/ NEJMra061808.

8. Zhang WW, Zhan SH, Geng CX, Sun X, Erkan M, Kleeff J, Xie XJ. Activated leukocyte cell adhesion molecule regulates the interaction between pancreatic cancer cells and stellate cells. Mol Med Rep. 2016; 14:3627-33. doi: 10.3892/mmr.2016.5681.

9. Weidle UH, Eggle D, Klostermann S, Swart GW. ALCAM/CD166: cancer-related issues. Cancer Genomics Proteomics. 2010; 7:231-43.

10. Hansen AG, Swart GW, Zijlstra A. ALCAM: Basis Sequence: Mouse. AFCS Nat Mol Pages. 2011; 2011. doi: 10.1038/mp.a004126.01.

11. Thelen K, Jaehrling S, Spatz JP, Pollerberg GE. Depending on its nano-spacing, ALCAM promotes cell attachment and axon growth. PLoS One. 2012; 7:e40493. doi: 10.1371/ journal.pone.0040493.

12. Han ZD, He HC, Bi XC, Qin WJ, Dai QS, Zou J, Ye YK, Liang YX, Zeng GH, Zhu G, Chen ZN, Zhong WD. Expression and clinical significance of CD147 in genitourinary carcinomas. J Surg Res. 2010; 160:260-7. doi: 10.1016/j.jss.2008.11.838.

13. Kristiansen G, Pilarsky C, Wissmann C, Stephan C, Weissbach L, Loy V, Loening S, Dietel M, Rosenthal A. ALCAM/CD166 is up-regulated in low-grade prostate cancer and progressively lost in high-grade lesions. Prostate. 2003; 54:34-43. doi: 10.1002/pros.10161.

14. King J, Ofori-Acquah S, Stevens T, Al-Mehdi AB. Potential role for activated leukocyte cell adhesion molecule and neural cadherin in metastasis to the lung microcirculation. Chest. 2004; 125:150S-1S.

15. Mezzanzanica D, Fabbi M, Bagnoli M, Staurengo S, Losa M, Balladore E, Alberti P, Lusa L, Ditto A, Ferrini S, Pierotti MA, Barbareschi M, Pilotti S, et al. Subcellular localization of activated leukocyte cell adhesion molecule is a molecular predictor of survival in ovarian carcinoma patients. Clin Cancer Res. 2008; 14:1726-33. doi: 10.1158/1078-0432.CCR-07-0428.

16. Kahlert C, Weber H, Mogler C, Bergmann F, Schirmacher P, Kenngott HG, Matterne U, Mollberg N, Rahbari NN, Hinz U, Koch M, Aigner M, Weitz J. Increased expression of ALCAM/CD166 in pancreatic cancer is an independent prognostic marker for poor survival and early tumour relapse. Br J Cancer. 2009; 101:457-64. doi: 10.1038/ sj.bjc.6605136.

17. Farhana L, Antaki F, Anees MR, Nangia-Makker P, Judd S, Hadden T, Levi E, Murshed F, Yu Y, Van Buren E, Ahmed K, Dyson G, Majumdar AP. Role of cancer stem cells in racial disparity in colorectal cancer. Cancer Med. 2016; 5:1268-78. doi: 10.1002/cam4.690.

18. Moslemi D, Mohammadianpanah M. Association of CD166 expression with clinicopathologic characteristics of colorectal cancer. Caspian J Intern Med. 2016; 7:150.

19. Sim SH, Kang MH, Kim YJ, Lee KW, Kim DW, Kang SB, Eom KY, Kim JS, Lee HS, Kim JH. P21 and CD166 as predictive markers of poor response and outcome after fluorouracil-based chemoradiotherapy for the patients with rectal cancer. BMC Cancer. 2014; 14:241. doi: 10.1186/1471-2407-14-241.

20. Zhou J, Belov L, Chapuis P, Chan C, Armstrong N, Kaufman KL, Solomon MJ, Clarke SJ, Christopherson RI. Surface profiles of live colorectal cancer cells and tumor infiltrating lymphocytes from surgical samples correspond to prognostic categories. J Immunol Methods. 2015; 416:59-68. doi: 10.1016/j.jim.2014.11.001.

21. Tachezy M, Zander H, Gebauer F, Marx A, Kaifi JT, Izbicki JR, Bockhorn M. Activated leukocyte cell adhesion molecule (CD166) - its prognostic power for colorectal cancer patients. J Surg Res. 2012; 177:e15-20. doi: 10.1016/j.jss.2012.02.013.

22. Lugli A, Iezzi G, Hostettler I, Muraro MG, Mele V, Tornillo L, Carafa V, Spagnoli G, Terracciano L, Zlobec I. Prognostic impact of the expression of putative cancer stem cell markers CD133, CD166, CD44s, EpCAM, and ALDH1 in colorectal cancer. Br J Cancer. 2010; 103:382-90. doi: 10.1038/sj.bjc.6605762.

23. Lee HJ, Eom DW, Kang GH, Han SH, Cheon GJ, Oh HS, Han KH, Ahn HJ, Jang HJ, Han MS. Colorectal micropapillary carcinomas are associated with poor prognosis and enriched in markers of stem cells. Mod Pathol. 2013; 26:1123-31. doi: 10.1038/modpathol.2012.163.

24. Weichert W, Knosel T, Bellach J, Dietel M, Kristiansen G. ALCAM/CD166 is overexpressed in colorectal carcinoma and correlates with shortened patient survival. J Clin Pathol. 2004; 57:1160-4. doi: 10.1136/jcp.2004.016238.

25. Horst D, Kriegl L, Engel J, Kirchner T, Jung A. Prognostic significance of the cancer stem cell markers CD133, CD44, and CD166 in colorectal cancer. Cancer Invest. 2009; 27:844-50. doi: 10.1080/07357900902744502.

26. Ni C, Zhang Z, Zhu X, Liu Y, Qu D, Wu P, Huang J, Xu AX. Prognostic value of CD166 expression in cancers of the digestive system: a systematic review and meta-analysis. PLoS One. 2013; 8: e70958. doi: 10.1371/journal.pone.0070958.

27. Burkhardt M, Mayordomo E, Winzer KJ, Fritzsche F, Gansukh T, Pahl S, Weichert W, Denkert C, Guski H, 
Dietel M, Kristiansen G. Cytoplasmic overexpression of ALCAM is prognostic of disease progression in breast cancer. J Clin Pathol. 2006; 59:403-9. doi: 10.1136/ jcp.2005.028209.

28. van den Brand M, Takes RP, Blokpoel-deRuyter M, Slootweg PJ, van Kempen LC. Activated leukocyte cell adhesion molecule expression predicts lymph node metastasis in oral squamous cell carcinoma. Oral Oncol. 2010; 46:393-8. doi: 10.1016/j.oraloncology.2010.03.001.

29. Ross JS, Torres-Mora J, Wagle N, Jennings TA, Jones DM. Biomarker-based prediction of response to therapy for colorectal cancer: current perspective. Am J Clin Pathol. 2010; 134:478-90. doi: 10.1309/AJCP2Y8KTDPOAORH.

30. Walther A, Johnstone E, Swanton C, Midgley R, Tomlinson I, Kerr D. Genetic prognostic and predictive markers in colorectal cancer. Nat Rev Cancer. 2009; 9:489-99. doi: $10.1038 / \mathrm{nrc} 2645$.

31. Xu L, Mohammad KS, Wu H, Crean C, Poteat B, Cheng Y, Cardoso AA, Machal C, Hanenberg H, Abonour R, Kacena MA, Chirgwin J, Suvannasankha A, et al. Cell Adhesion Molecule CD166 Drives Malignant Progression and Osteolytic Disease in Multiple Myeloma. Cancer Res. 2016; 7:6901-6910. doi: 10.1158/0008-5472.CAN-16-0517.

32. Wachowiak R, Mayer S, Kaifi J, Gebauer F, Izbicki JR, Lacher M, Bockhorn M, Tachezy M. Prognostic Impact of Activated Leucocyte Cell Adhesion Molecule (ALCAM/ CD166) in Infantile Neuroblastoma. Anticancer Res. 2016; 36:3991-5.

33. Jia G, Wang X, Yan M, Chen W, Zhang P. CD166-mediated epidermal growth factor receptor phosphorylation promotes the growth of oral squamous cell carcinoma. Oral Oncol. 2016; 59:1-11. doi: 10.1016/j.oraloncology.2016.05.010.

34. Moon BS, Jeong WJ, Park J, Kim TI, Min do S, Choi KY. Role of oncogenic K-Ras in cancer stem cell activation by aberrant Wnt/beta-catenin signaling. J Natl Cancer Inst. 2014; 106:djt373. doi: 10.1093/jnci/djt373. 